\title{
Painful, swollen elbow
}

\section{Was a recent procedure responsible for this patient's pain?}

A 32-YEAR-OLD WOMAN presented to our clinic with left elbow swelling and pain of 6 days' duration. She'd had a posterior interosseous nerve (PIN) injection (hydrodissection) at another facility 12 days earlier for refractory intersection syndrome.

During nerve hydrodissection, fluid is injected into the area surrounding the nerve in an effort to displace the muscles, tendons, and fascia and thus reduce friction on the nerve. This treatment, often completed with ultrasound guidance, is utilized by patients who want to obtain pain relief without undergoing surgery for nerve entrapment syndromes.

In this case, a combination of $1 \mathrm{~mL}$ (40 mg) of methylprednisolone acetate, $1 \mathrm{~mL}$ of lidocaine $2 \%$, and $3 \mathrm{~mL}$ of normal saline was injected into the supinator muscle belly (proximal dorsal aspect of the forearm) under ultrasound guidance. Six days later, the patient began to experience elbow pain, redness, and swelling. The symptoms progressed within several hours and became so notable that she sought care at an urgent care facility the next morning. At this facility, she was told she had an infection and was prescribed oral levofloxacin $500 \mathrm{mg} / \mathrm{d}$.

The patient presented to our clinic after 4 days of oral levofloxacin with no improvement of symptoms. She denied chills or fever and described her pain as moderate and radiating to her fingers. There was no history of trauma. The patient reported riding her bike more frequently, which had caused the original forearm pain that warranted the PIN injection. There were no other recent changes to activity. Her medical, social, and surgical histories were otherwise unremarkable.

Her vital signs were normal. Physical exam revealed an erythematous and warm left elbow (FIGURE 1). Her left elbow range of motion (extension and flexion) was mildly decreased due to the pain and swelling.

\section{O WHAT IS YOUR DIAGNOSIS? \\ O HOW WOULD YOU TREAT THIS PATIENT?}

Morteza Khodaee, MD, MPH; Stephen Spadafore, MD; Jack Spittler, MD, MS Department of Family Medicine \& Orthopedics, University of Colorado School of Medicine, Denver

\section{- morteza.khodaee@ cuanschutz.edu}

DEPARTMENT EDITOR Richard P. Usatine, MD University of Texas Health at San Antonio

The authors reported no potential conflict of interest relevant to this article.

\section{FIGURE 1}

\section{Erythematous left elbow}
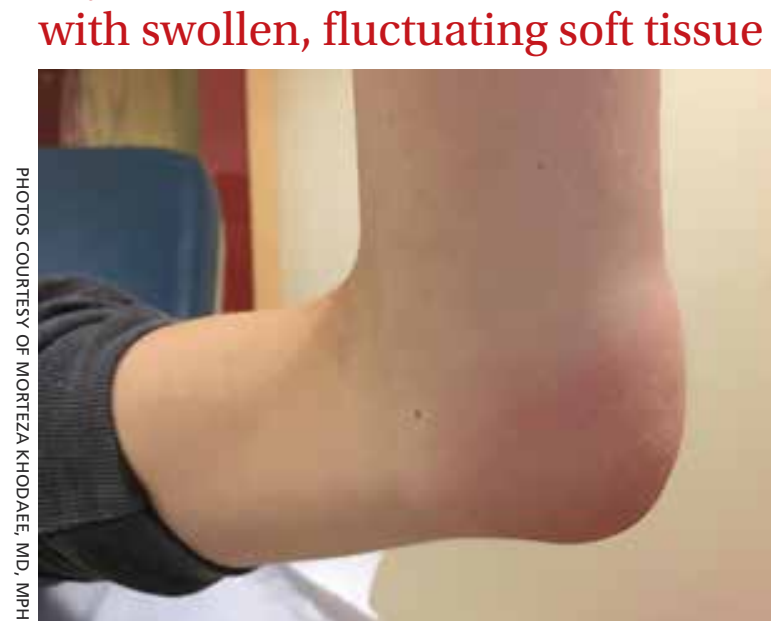


\section{$>$}

When septic
bursitis is
strongly
suspected or
confirmed, the
patient should
be started on
an antibiotic
regimen that
covers $S$ aureus.

\section{Diagnosis:}

latrogenic septic olecranon bursitis Aspiration of the patient's olecranon bursa produced $3 \mathrm{~mL}$ of cloudy fluid (FIGURE 2). The patient's painful, swollen, erythematous, warm elbow, cloudy aspirate, and history of preceding PIN hydrodissection were consistent with a diagnosis of septic olecranon bursitis.

Septic bursitis usually is caused by bacteria. ${ }^{1,2}$ Bursal infection can result from the spread of infection from nearby tissues or direct inoculation from skin trauma. It can also be iatrogenic and occur among healthy individuals. $^{2,3}$ Injection anywhere close to the bursa can inoculate enough bacteria to progress to cellulitis first and then septic bursitis. Inflammatory conditions such as gout and rheumatoid arthritis also can cause acute and/or chronic superficial bursitis. $^{1,2,4}$

Differentiating between septic and nonseptic bursitis can be challenging on his-

\section{FIGURE 2}

\section{Bursal fluid extracted from the site}

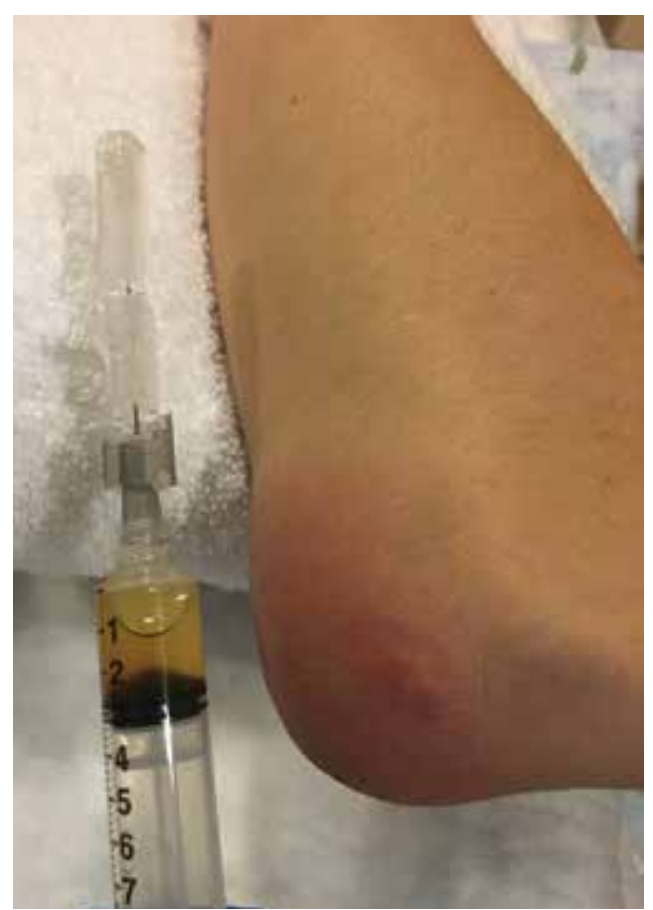

Under ultrasound guidance, $3 \mathrm{~mL}$ of cloudy bursal fluid was aspirated. tory and physical exam alone, but specific signs and symptoms should warrant concern for infection. ${ }^{1,2,45}$ Fever is present in up to $75 \%$ of septic cases ${ }^{5}$; however, lack of fever does not rule out septic bursitis. Pain, erythema, warmth, and an overlying skin lesion also can indicate infection. ${ }^{4}$ Diagnostic imaging modalities may help distinguish different types of olecranon bursitis, but in most cases, they are not necessary. ${ }^{2}$

\section{Other joint disorders \\ factor into the differential}

The differential diagnosis is broad and includes a variety of joint disorders in addition to septic (and nonseptic) bursitis., ${ }^{2,3}$

Septic arthritis is a deeper infection that involves the elbow joint and is considered an orthopedic emergency due to potential joint destruction.

A simple joint effusion also arises from the elbow joint, but this diagnosis becomes less likely when the joint aspirate appears cloudy. A simple joint effusion would not produce bacteria on gram stain and culture.

Crystalline inflammatory arthritis (gout, pseudogout) is due to intra-articular precipitation of crystals (uric acid crystals in gout, calcium pyrophosphate crystals in pseudogout).

- Hematomas would produce gross blood or clot on joint aspiration.

Cellulitis is an infection of the superficial soft tissue (only) and thus, aspiration is not likely to yield fluid.

\section{Diagnosis can be made with culture of fluid}

Confirmation of septic olecranon bursitis is best attained by bursal needle aspiration and culture. Aspiration also can evaluate for other causes of elbow swelling. (If septic olecranon bursitis is suspected clinically, empiric antibiotics should be started while awaiting culture results. ${ }^{6}$ ) White blood cell counts from the aspirate also may be utilized but have a lower sensitivity and specificity for diagnosis. ${ }^{7}$

In addition to aiding in diagnosis, bursal aspiration for a patient with septic bursitis can improve symptoms and reduce bacterial load. ${ }^{1-3,8}$ The use of a compressive bandage after aspiration may help reduce re-accumu- 
lation of the bursal fluid..$^{1-3,8}$ Staphylococcus aureus is responsible for the majority of septic olecranon bursitis cases. ${ }^{9-11}$

\section{Tailoring the antibiotic regimen}

There is wide variation in the treatment of septic olecranon bursitis due to the lack of strong evidence-based guidelines. ${ }^{1,2,8,11-13}$ When septic bursitis is strongly suspected (or confirmed) the patient should be started on an antibiotic regimen that covers $S$ aureus. ${ }^{1,2}$ Once culture results and sensitivities return, the antibiotic regimen can be tailored appropriately.

In cases of mild-to-moderate septic olecranon bursitis in an immunocompetent host, the patient can be started on oral antibiotics and monitored closely as an outpatient. ${ }^{1-3,8}$ Patients with septic olecranon bursitis who meet the criteria for systemic inflammatory response syndrome or who are immunocompromised should be hospitalized and started on intravenous antibiotics. ${ }^{1-3}$ Recommended duration of antibiotic therapy varies but is usually about 10 to 14 days..$^{1-3,8}$ In rare cases, surgical intervention with bursectomy may be necessary. ${ }^{1,2,14}$

- Our patient was given a dose of ceftriaxone $250 \mathrm{mg}$ intramuscularly and was started on oral sulfamethoxazole/trimethoprim $800 \mathrm{mg} / 160 \mathrm{mg}$ twice daily after aspiration of the bursa. Culture of the bursal fluid grew oxacillin-sensitive $S$ aureus which was sensitive to a variety of antibiotics including levofloxacin and sulfamethoxazole/trimethoprim. Her symptoms gradually improved (FIGURE 3) and resolved after a 14-day course of oral sulfamethoxazole/trimethoprim.

JFP

CORRESPONDENCE

Morteza Khodaee, MD, MPH, University of Colorado School of Medicine, Department of Family Medicine \& Orthopedics, AFW Clinic, 3055 Roslyn St, Denver, CO 80238; morteza. khodaee@cuanschutz.edu

\section{References}

1. Baumbach SF, Lobo CM, Badyine I, et al. Prepatellar and olecranon bursitis: literature review and development of a treatment

\section{FIGURE 3}

\section{Improvement of the erythema} and swelling

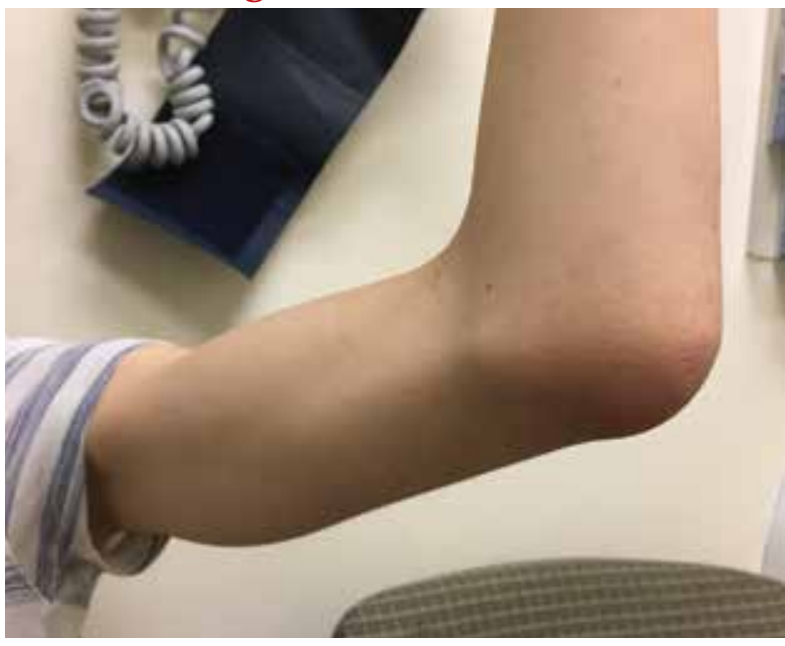

Improvement of the left elbow erythema and swelling was noted 10 days after initiating treatment with oral sulfamethoxazole/ trimethoprim.

algorithm. Arch Orthop Trauma Surg. 2014;134:359-370.

2. Khodaee M. Common superficial bursitis. Am Fam Physician. 2017;95:224-231.

3. Harris-Spinks C, Nabhan D, Khodaee M. Noniatrogenic septic olecranon bursitis: report of two cases and review of the literature. Curr Sports Med Rep. 2016;15:33-37.

4. Reilly D, Kamineni S. Olecranon bursitis. J Shoulder Elbow Surg. 2016;25:158-167.

5. Blackwell JR, Hay BA, Bolt AM, et al. Olecranon bursitis: a systematic overview. Shoulder Elbow. 2014;6:182-190.

6. Del Buono A, Franceschi F, Palumbo A, et al. Diagnosis and management of olecranon bursitis. Surgeon. 2012;10:297-300.

7. Stell IM, Gransden WR. Simple tests for septic bursitis: comparative study. BMJ. 1998;316:1877.

8. Abzug JM, Chen NC, Jacoby SM. Septic olecranon bursitis. J Hand Surg Am. 2012;37:1252-1253.

9. Cea-Pereiro JC, Garcia-Meijide J, Mera-Varela A, et al. A comparison between septic bursitis caused by Staphylococcus aureus and those caused by other organisms. Clin Rheumatol. 2001;20:10-14.

10. Morrey BE. Bursitis. In: Morrey BE, Sanchez-Sotelo J, eds. The Elbow and its Disorders. 4th ed. Philadelphia, PA: Saunders Elsevier 2009:1164-1173

11. Wingert NC, DeMaio M, Shenenberger DW. Septic olecranon bursitis, contact dermatitis, and pneumonitis in a gas turbine engine mechanic. J Shoulder Elbow Surg. 2012;21:E16-E20.

12. Baumbach SF, Michel M, Wyen H, et al. Current treatment concepts for olecranon and prepatellar bursitis in Austria. Z Orthop Unfall. 2013;151:149-155.

13. Sayegh ET, Strauch RJ. Treatment of olecranon bursitis: a systematic review. Arch Orthop Trauma Surg. 2014;134:1517-1536.

14. Ogilvie-Harris DJ, Gilbart M. Endoscopic bursal resection: the olecranon bursa and prepatellar bursa. Arthroscopy. 2000;16:249253.

\section{(2) Visit us @ mdedge.com/familymedicine}

